\title{
Redundancy Dependence in the Context of Competing Risks and Dynamic Degradation
}

\author{
Tom Van Acker, Member, IEEE, and Dirk Van Hertem, Senior Member, IEEE
}

\begin{abstract}
Parallel redundancy is a common approach to increase network availability. As a consequence of providing redundancy, the loading of that component changes, affecting its reliability. A holistic approach combining two models is introduced to assess both instantaneous and future effects on general failure rates in the context of competing risks and dynamic degradation. Concretely, both are designed to account for the duration of abnormal loading events. First, a per-unitdegradation method is introduced to map the different loading states onto a single normal operation sojourn time variable. Second, a time-inhomogeneous Markov chain is introduced to assess the irreversible degradation following a nondetrimental abnormal loading event. The numerical illustration shows that both models are necessary to correctly assess the effects of redundancy dependence if the underlying failure distribution has a shape parameter exceeding one.
\end{abstract}

Index Terms-Availability, degradation, Markov process, multistate model, redundancy dependence.

\section{NOMENCLATURE}

\begin{tabular}{|c|c|}
\hline$\alpha$ & degradation \\
\hline$t$ & calendar time \\
\hline $\mathcal{A}$ & set of cohort \\
\hline $\mathcal{F}$ & set of all failures \\
\hline $\mathcal{F}^{\mathrm{w}}$ & subset of wear-out failures $\left(\mathcal{F}^{\mathrm{w}} \subset \mathcal{F}\right)$ \\
\hline $\mathcal{L}$ & $\begin{array}{l}\text { set of loading states: union of normal and } \\
\text { abnormal loading states }\left(=\mathcal{L}^{\mathrm{n}} \cup \mathcal{L}^{\mathrm{a}}\right)\end{array}$ \\
\hline $\mathcal{S}$ & $\begin{array}{l}\text { set of component states: union of normal operation } \\
\text { and post-failure states }(=\mathcal{N} \cup \mathcal{P})\end{array}$ \\
\hline$\left(x, \varphi_{x}\right)$ & $\begin{array}{l}\text { semicontinuous state, where } x \text { represents the state } \\
\text { itself and } \varphi_{x} \text { its sojourn time. }\end{array}$ \\
\hline$p_{x}$ & state probability \\
\hline$\hat{p}_{x}$ & state entry probability \\
\hline $\mathfrak{f}$ & probability density function \\
\hline$R$ & survival function \\
\hline$\lambda$ & failure rate \\
\hline$\Delta \alpha^{\text {rel }}$ & relative degradation function \\
\hline
\end{tabular}

\section{INTRODUCTION}

\section{A. Background and Motivation}

I $\mathrm{N}$ his 2009 review paper [1], Enrico Zio indicated modeling complexity of operation dependencies in multistate systems (MSS) as one of the major challenges in reliability engineering. Dependence describes the quality of an item being influenced by (a system of) other items, and subcategories

T. Van Acker and D. Van Hertem are with the Departement of Electrical Engineering, KU Leuven, Leuven, Belgium (e-mail: tom.vanacker@esat.kuleuven.be; dirk.vanhertem@esat.kuleuven.be).

This research is funded by a Ph.D. Fellowship of the Flemish Agency for Innovation \& Entrepreneurship in cooperation with BASF Antwerp NV (Grant No. 150257). include: economic, structural and stochastic dependence. This paper focuses on stochastic dependence, describing the effects of change in principal random variables on the conditional distribution of dependent random variables ${ }^{1}$. Stochastic dependence may either be positive or negative, i.e., the conditional probability of a dependent random variable is respectively strictly higher or lower given the change of its principal.

Parallel redundancy is a common approach to increase network availability, introducing a specific form of stochastic dependence: redundancy dependence, either active, e.g., loadsharing systems, or passive redundancy. Redundancy dependence introduces a failure dependence between different components with redundancy options, as the reliability of certain components depends on the state-space of others. When providing redundancy, the loading of those components changes, affecting their reliability in two ways: (i) stress, affecting instantaneous failure rates; and (ii) degradation, affecting future failure rates. Degradation is defined as detrimental change in the ability to meet requirements [2]. For example, the paper insulation of an oil-immersed transformer degrades depending on thermal stress, reducing its ability to isolate different winding layers and eventually leading to its failure. Network component degradation may have different mechanisms: chemical, electrical, mechanical and thermal, each of which is dependent on different drivers such as weather, loading, etc. Except for the loading changes, most of these degradation drivers are extrinsic. Furthermore, the degradation level depends on the duration for which a component is exposed to these load changes. Expanding on the previous example, the accelerated reduction in the lifetime of an oilimmersed transformer is dependent on both the increase in load, i.e., the hot-spot temperature, and the event duration [3].

In a competing risk context, each with their respective maintainability and recoverability actions, the unavailability duration of the principal component diversifies. Consequently, the degradation of the dependent component providing redundancy also diversifies. To account of these diverse degradation effects, it becomes pertinent to frame redundancy dependence in the context of competing risks with general failure rates to enable account for different abnormal loading durations.

\section{B. Redundancy Dependence Literature Review}

A lot of work has been done on this subject, however, the effects of loading duration on component degradation in the context of general failure rates have not yet been studied, although they are highly relevant in reality. Early work on

\footnotetext{
${ }^{1}$ Notation: a principal causes the effect on its dependent
} 
multicomponent systems mostly assumed independent components. Although such an assumption allows for tractable models, it might induce significant errors where dependence is present. The well-known '91 special issue on stochastic dependence bundled some early work on the topic, mostly focussing on cascading failures, negative failure dependence and common cause failures. Recently, redundancy dependence has experienced increased attention in the literature.

Kotz et al. were amongst the first to show the effects of redundancy dependence on the mean lifetime of two component systems [4]. In this work, the correlation effects are modeled through the concept of quadrant dependence. Blockus presents a limit reliability function for large parallel-series systems, where a single component lifetime distribution is assumed to be Markovian and scaled with the number of remaining parallel components [5]. Extending on the work of Blockus, Yu et al. present a classification of redundant dependency within the context of reliability optimization, still under the assumption of Markovian lifetime distributions [6]. Costa Bueno derived a closed form solution for redundancy allocation in $k$-out-of- $n: \mathrm{F}$ systems using a martingale approach, both for standby [7] and active redundancy [8]. Extending on the work of Costa Bueno, Belzunce et al. present a closed form solution for redundancy allocation when all components are dependent [9].

All these works consider systems with non-repairable components, i.e., their common goal is to determine system reliability rather than its availability. To this end, Yu et al. extend their previous work by allowing perfect maintenance following a component failure, the corresponding rate of the latter is still assumed to be Markovian [10]. Another extension by Yuan presents a similar approach for a $k$-out-of- $n: G$ system with limited repair capacity given repairmen having multiple vacations [11]. Norros et al. consider three approaches to model repairable systems with dependent components by a multivariate stochastic on-off process [12].

All these works assume that following a non-fatal stress event no remnant effects of the additional stress remain. To this end, Jia et al. present a reliability evaluation approach based on multistate decision diagram for demand-based warm standby systems considering a degradation process [13]. Recently, $\mathrm{Xu}$ et al. classified extant forms of stochastic failure dependence considering degradation in multicomponent systems [14] into:

1) rate-rate: dependence between failure rates, e.g., Sun et al. present an analytical approach to account for rate-rate dependence in multicomponent systems through the concept of interacting failures [15].

2) state-rate: dependence between discrete degradation states and failure rates, e.g., Wang et al. present a multistate Markov system where its failure rates are dependent on a two-dimensional vector which describes the condition of its components [16].

3) degradation-rate: dependence between continuous degradation and failure rates, e.g., Caballé et al. use a nonhomogeneous Poisson process and gamma process to respectively model degradation starting times and growth. Furthermore, a doubly stochastic Poisson process is used to model detrimental shocks [17].

4) shock-degradation: dependence of failure rates on both discrete shocks and continuous degradation, e.g., Fan et al. model the shock-degradation dependence by assuming that the intensity function of the nonhomogeneous Poisson process describing the random shock processes is dependent on the degradation processes. Furthermore, a Monte Carlo approach is presented to calculate the system reliability [18].

\section{Contributions and Organization of the Paper}

However, none of the papers enumerated in the previous subsection holistically capture the intended goal of this paper with respect to redundancy dependence. This paper considers the effects of redundancy dependence through the concept of degradation, accounting for:

1) competing risks, each with their respective maintainability and recoverability actions;

2) general failure rates, dependent on both calendar time and state sojourn time; and

3) dynamic degradation, where the duration of an abnormal loading event impact the resulting degradation.

In prior work by the authors, a stochastic model was presented for the availability assessment of a component [19]. To allow for general transition rates and different restoration degrees, the state space is considered to be semicontinuous, including any state' sojourn time. In this paper, these sojourn times are exploited as they also may be used to describe the duration of providing redundancy. However, direct accounting for redundancy dependence in such a framework would require considering the dependent components as one system, expanding the dimension of the state' sojourn time vector. This would greatly decrease computational tractability. To avoid this, a system is split in its components, which are evaluated separately, and their availability is used as input to model the redundancy dependence. To this end, this paper presents two models:

1) a per-unit-degradation method to model the instantaneous effects of multiple loading conditions on general failure rates in the context of competing risks; and

2) a time-inhomogeneous Markov chain to assess the irreversible degradation following nondetrimental stress operation for a certain duration.

The contribution of this paper is the combination of both models in the context of a multistate semicontinuous statespace, as to holistically capture the effects of competing risks, general failure rates and dynamic degradation.

The remainder of the paper is organized as follows: Section II presents a mathematical framework which enables accounting for redundancy dependence and associated degradation. Section III introduces a numerical illustration to demonstrate the effects of redundancy dependence on the unavailability of a transformer. Finally, Section IV concludes the paper. Although this paper focusses on redundancy dependence, the presented models are also applicable to other forms of stochastic dependence, e.g., negative failure dependence for multiple series-connected components covered by the same protection system. 


\section{MATHEMATiCAL FRAMEWORK}

This section presents two models to account for redundancy dependence between components, incorporating the effect of abnormal loading duration. First, the concept of loading states is introduces in the context of the multistate semicontinuous state-space discussed in [19]. Next, a per-unit-degradation approach is presented to model the effects of multiple loading conditions on instantaneous failure rates. Lastly, a timeinhomogeneous Markov chain is presented to account for irreversible degradation following nondetrimental abnormal loading conditions, affecting future failure rates.

\section{A. Loading States in a Semicontinuous Multistate Context}

A stochastic model is presented in [19] for the availability assessment of a single component. It assumes a semicontinuous state-space, meaning that the state description for any state $x$ is extended with its sojourn time $\varphi_{x}$ resulting in $\left(x, \varphi_{x}\right)$. The state sojourn time $\varphi_{x}$ is the amount of time spent in a state $x$ before leaving that state. Furthermore, a multistate state-space is considered, i.e., a component is allowed to have an arbitrary (finite) number of states [20]. This enables accounting for competing risks, each with distinct maintainability and recoverability actions. Specifically, the state-space $\mathcal{S}$ is composed of one normal operation state $\left(n, \varphi_{n}\right) \in \mathcal{N}$ and multiple post-failure states $\left(p, \varphi_{p}\right) \in \mathcal{P}$. A transition set $\mathcal{T}$ which describes transitions $i j$ between states $\left(i, \varphi_{i}\right),\left(j, \varphi_{j}\right) \in \mathcal{S}$. All transitions $i j \in \mathcal{T}$ except for the failure transitions $n j$ are described by a weighted $\operatorname{pdf} \mathfrak{f}_{i j}\left(\varphi_{i}\right)$, depending on the current state sojourn time $\varphi_{i}$.

In [19], the system conditions are assumed to be known a priori, and therefore so are its failure rates. However, this assumption no longer holds if the loading of one component may become dependent on the state of others, affecting its reliability. Direct accounting for such dependencies using the stochastic process presented in [19] would require modeling all dependent components as a single system. As each component has its own normal operation sojourn time $\varphi_{n}$, this would change the normal operation state $\left(n, \varphi_{n}\right)$ into $\left(n, \varphi_{n}\right)$. However, as computational effort increases exponentially with the dimension of the underlying problem space, i.e., $\operatorname{dim}\left(\boldsymbol{\varphi}_{n}\right)+1$, such a direct approach is inadvisable ${ }^{2}$. In this section, a method is presented to decouple the problem in individual components, assessing each component individually, and modeling their redundancy dependence through loading states. Besides the advantage in computational efficiency, the approach enables accounting for duration of stress events, utilizing the state sojourn times of the principal components.

A specific component $\phi$ is only subject to a limited set of loading states $\mathcal{L}_{\phi}$, describing the number of end users supplied through that component $\phi$. The loading state set $\mathcal{L}_{\phi}$ may be classified into two subsets:

1) a singular subset $\mathcal{L}_{\phi}^{\mathrm{n}}$ corresponding to the normal loading condition, e.g., Fig. 1a; and

2) a subset $\mathcal{L}_{\phi}^{\mathrm{a}}=\mathcal{L}_{\phi} / \mathcal{L}_{\phi}^{\mathrm{n}}$ corresponding to all abnormal loading conditions, e.g., Fig. 1b.

\footnotetext{
${ }^{2}$ The additional dimension is related to the calendar time $t$.
}

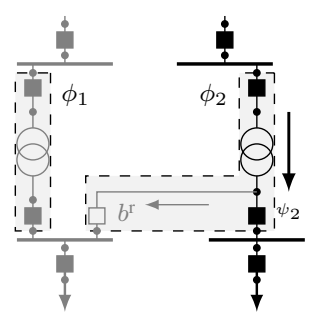

(a) normal loading $l_{1} \in \mathcal{L}_{\phi_{2}}^{\mathrm{n}}$

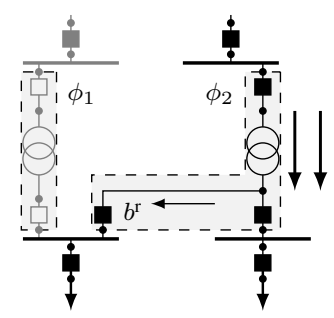

(b) abnormal loading $l_{2} \in \mathcal{L}_{\phi_{2}}^{\mathrm{a}}$
Fig. 1. Possible loading states of the dependent component $\phi_{2}$ : (a) normal loading, and (b) abnormal loading.

Any loading state $\left(l, \varphi_{l}\right) \in \mathcal{L}_{\phi}$ is discrete with respect to the loading state $l$ itself but continuous with respect to its sojourn time $\varphi_{l}$. The latter essentially represents the duration for which the component has been in that specific loading condition. The stochastic process $L(t, \varphi)$ describes the loading state of a dependent component $\phi$ with respect to time $t$ and duration $\varphi$, and is dependent on the state-space of the principal component. More specifically, a loading state $\left(l, \varphi_{l}\right)$ of a dependent component corresponds with a subset of states $\left(s, \varphi_{s}\right) \in \mathcal{S}$ of its principal component. A loading state $\left(l, \varphi_{l}\right)$ is fully defined by the following measures:

1) $p_{l}(t)$ : the probability that a component is in specified loading state $l$ at time instant $t$;

2) $\hat{p}_{l}(t)$ : the probability that a component enters a specified loading state $l$ at time instant $t$; and

3) $\mathfrak{f}_{l}\left(\varphi_{l}\right)$ : the probability density function of the loading state sojourn time $\varphi_{l}$.

In a general setting, a loading state may be entered in multiple ways, resulting in vectors $\hat{\boldsymbol{p}}_{l}(t)$ and $\mathfrak{f}_{l}\left(\varphi_{l}\right)$. To put this into context, the necessary measures for the dependent component $\phi_{2}$ in Fig. 1 are derived from the state-transition diagram of its principal component $\phi_{1}$ (Fig. 2). The principal component $\phi_{1}$ is subject to a single failure $f$, corresponding with the transition from $\left(n, \varphi_{n}\right)$ to fault state $\left(f, \varphi_{f}\right)$, and has two protection zones, each corresponding to the opening of different interruptors, respectively leading to states $\left(r, \varphi_{r}\right)$ and $\left(s, \varphi_{s}\right)$. In this example, it is assumed that component $\phi_{2}$ provides redundancy for $\phi_{1}$ by closing breaker $b^{\mathrm{r}}$, only after a fault on the latter has been cleared, i.e., after exiting state $\left(f, \varphi_{f}\right)$. Given that a fully described state-space of the principal component $\phi_{1}$, the necessary loading state measures for the dependent component $\phi_{2}$ may be derived (Table I).

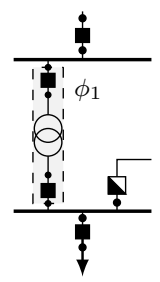

(a)

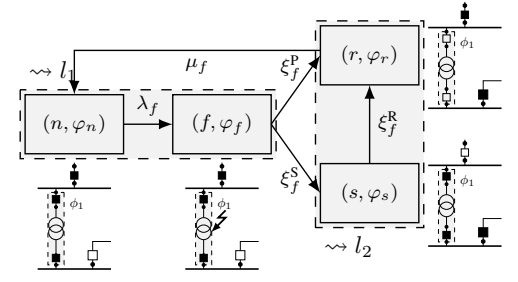

(b)
Fig. 2. Principal component $\phi_{1}$ in Fig. 1 (2a) and its state-transition diagram (2b) annotated to show $\mathcal{L}_{\phi_{2}}$. 
TABLE I

LOADING STATES MEASURES OF DEPENDENT COMPONENT $\phi_{2}$ (FIG. 1 ).

\begin{tabular}{l|lll} 
& $p_{l}(t)$ & $\hat{\boldsymbol{p}}_{l}(t)$ & $\mathfrak{f}_{l}\left(\varphi_{l}\right)$ \\
\hline$l_{1}$ & $p_{n}(t)+p_{f}(t)$ & {$\left[\hat{p}_{n}(t)\right]$} & {$\left[\mathfrak{f}_{\lambda_{f}} *\left(\mathfrak{f}_{\xi_{f}^{\mathrm{P}}}+\mathfrak{f}_{\xi_{f}^{\mathrm{P}}}\right)\right]$} \\
$l_{2}$ & $p_{r}(t)+p_{s}(t)$ & {$\left[\hat{p}_{r}(t), \hat{p}_{s}(t)\right]$} & {$\left[\mathfrak{f}_{\xi_{f}^{\mathrm{R}}} * \mathfrak{f}_{\mu_{f}}, \mathfrak{f}_{\mu_{f}}\right]$} \\
\hline
\end{tabular}

\section{B. Effects on Instantaneous Failure Rates}

In this subsection, a per-unit degradation approach is presented to handle the effects of a non-empty subset $\mathcal{L}_{\phi}^{\text {a }}$ on instantaneous failure rates. Any component $\phi$ is subject to a set of failures $\mathcal{F}_{\phi}$ with corresponding general failure rates $\lambda_{f}\left(t, \varphi_{n} ; L\right)$, only when in its normal operation state $\left(n, \varphi_{n}\right)$. A component's normal operation time $\varphi_{n}$ relates to calendar time $t$ through a variable $a: a=t-\varphi_{n}$, i.e., the calendar time at which the component was installed. In mathematical biology, the variable $a$ is referred to as a cohort [21]. In the context of this paper, a discrete cohort set $\mathcal{A} \subset \mathbb{Z}$ is introduced. Each cohort $a$ groups the probability of components which were installed within a timeframe $t \in[a \mathrm{~d} a,(a+1) \mathrm{d} a]$, where $\mathrm{d} a$ denotes the intercohort step size. For each cohort $a \in \mathcal{A}$, any failure rate $\lambda_{f}\left(t, \varphi_{n} ; L\right)$ may be reformulated as $\lambda_{a, f}\left(\varphi_{n} ; L\right)$. Furthermore, loading state measures $p_{l}(t)$ and $\hat{p}_{l}(t)$ may be translated into respectively $p_{a, l}\left(\varphi_{n}\right)$ and $\hat{p}_{a, l}\left(\varphi_{n}\right)$.

The different failures are modeled through the concept of competing risk [22]. Competing risks are said to be present when: (a) a component is at risk of more than one mutually exclusive failure; and (b) the occurrence of any one prevents any other from happening. This definition holds within the context of this paper as: (a) all loading states are mutually exclusive and therefore also the corresponding failure rates, as the latter is the direct consequence of the former; and (b) any failure on a certain cohort $a$ results in the departure from that cohort, ensuring no other failure can occur along that cohort $a$. The function $\lambda_{a, f}\left(\varphi_{n} ; L\right)$ gives the instantaneous failure rate from a failure $f$ along a cohort $a$ as

$$
\begin{aligned}
& \lambda_{a, f}\left(\varphi_{n} ; L\right)= \\
& \quad \lim _{\Delta \varphi_{n} \rightarrow 0} \frac{\mathbb{P}\left(\varphi_{n} \leq \Phi_{n}<\varphi_{n}+\Delta \varphi_{n}, \mathcal{A}=a, \mathcal{F}=f \mid \Phi_{n} \geq \varphi_{n} ; L\right)}{\Delta \varphi_{n}},
\end{aligned}
$$

where $\Phi_{n}$ represents the normal operation sojourn time to failure. The overall failure rate $\lambda_{a}\left(\varphi_{n} ; L\right)$ along a cohort $a$ is described by

$$
\lambda_{a}\left(\varphi_{n} ; L\right)=\sum_{f \in \mathcal{F}_{\phi}} \lambda_{a, f}\left(\varphi_{n} ; L\right) .
$$

A subset of failures $f \in \mathcal{F}_{\phi}^{\mathrm{w}} \subset \mathcal{F}_{\phi}$, referred to as wear-out failures, are dependent on the specific loading state $\left(l, \varphi_{l}\right) \in \mathcal{L}_{\phi}$. In its most rudimental form, the wear-out of a component $\phi$ is described by a pdf $\mathfrak{f}_{f}^{\mathrm{w}}(\alpha)$ with respect to that component's degradation $\alpha$. Depending on its loading, a component degrades quicker or slower with respect to its normal operation time $\varphi_{n}$, or in other words the normal operation time after which a component is expected to wear out depends on its loading. For example, a paper insulted transformer under loading conditions $\mathrm{I}^{\mathrm{pu}}=0.5$ and $\mathrm{I}^{\mathrm{pu}}=1.0$ is expected to wear out due to thermal degradation of the insulation paper after an operation time of respectively $5985.8 \mathrm{yr}$ and $83.5 \mathrm{yr}^{3}$. Given these different degradation speeds with respect to normal operation time $\varphi_{n}$, it becomes necessary to choose a basis with respect to which all loading conditions are scaled, i.e., the normal loading condition $l \in \mathcal{L}_{\phi}^{\mathrm{n}}$. In other words, it is assumed that a component degrades a single day for every day it spends in the normal loading condition. Consequently, the component wear-out may be modeled through a pdf $\mathfrak{f}_{f}^{\mathrm{w}}\left(\varphi_{n}\right)$ with the expected value corresponding to the normal loading condition, e.g., $5985.8 \mathrm{yr}$ if $\mathrm{I}^{\mathrm{pu}}=0.5$. In doing so, the wear-out failure corresponding to the normal loading condition $l \in \mathcal{L}_{\phi}^{\mathrm{n}}$ is fully described by

$$
\mathfrak{f}_{a, f}\left(\varphi_{n}\right)=p_{a, l}\left(\varphi_{n}\right) \mathfrak{f}_{f}^{\mathrm{w}}\left(\varphi_{n}\right), \quad \forall f \in \mathcal{F}_{\phi}^{\mathrm{w}}, f \rightarrow l \in \mathcal{L}_{\phi}^{\mathrm{n}} .(3)
$$

where $p_{a, l}\left(\varphi_{n}\right)$ represents the probability of being in a loading condition $l \in \mathcal{L}_{\phi}^{\mathrm{n}}$. In the case of a single loading condition, the loading probability $p_{a, l}\left(\varphi_{n}\right)$ would be equal to one, and the entire approach would simply fall under the discussion in [19]. However, entering an abnormal loading condition $l \in$ $\mathcal{L}_{\phi}^{\mathrm{a}}$ alters the stress on that component, and consequently alters the speed at which the pdf $\mathfrak{f}_{f}^{\mathrm{w}}$ is traversed. For example, a paper insulated transformer degrades 71.6 times faster in the loading condition $\mathrm{I}^{\mathrm{pu}}=1.0$ compared to the normal loading condition $\mathrm{I}^{\mathrm{pu}}=0.5$, and therefore traverses the wear-out pdf $\mathfrak{f}^{\mathrm{W}}$ 71.6 times faster. To this end, a loading specific $\operatorname{pdf} \mathfrak{f}_{l}^{\mathrm{w}}\left(\varphi_{n}\right)$ is constructed for all abnormal loading conditions $l \in \mathcal{L}_{\phi}^{\text {a }}$ with respect to the pdf $\mathfrak{f}_{f}^{\mathrm{w}}\left(\varphi_{n}\right)$ corresponding to the normal loading condition:

$$
\mathfrak{f}_{l}^{\mathrm{w}}\left(\varphi_{n}\right)=\int_{\varphi_{n}}^{\varphi_{n}+\mathbb{E}\left(\Delta \alpha_{l}^{\text {rel }}\right)} \mathfrak{f}_{f}^{\mathrm{w}}\left(\varphi_{x}\right) \mathrm{d} \varphi_{x}, \quad \forall f \in \mathcal{F}_{\phi}^{\mathrm{w}}, f \rightarrow l \in \mathcal{L}_{\phi}^{\mathrm{a}} .
$$

The expected relative degradation $\mathbb{E}\left(\Delta \alpha_{l}^{\mathrm{rel}}\right)$ associated with an abnormal loading condition $l \in \mathcal{L}_{\phi}^{\mathrm{a}}$ may be determined by combining the relative degradation function $\Delta \alpha_{l}^{\text {rel }}\left(\varphi_{l}\right)$ with the pdf $\mathfrak{f}_{l}\left(\varphi_{l}\right)$ representative of the probability that the component remains in the loading state $l$ for a duration $\varphi_{l}$. Using the loading specific pdf $\mathfrak{f}_{l}^{\mathrm{w}}\left(\varphi_{n}\right)$, the corresponding failure pdf $\mathfrak{f}_{a, f}\left(\varphi_{n}\right)$ may be determined as follows:

$$
\mathfrak{f}_{a, f}\left(\varphi_{n}\right)=\hat{p}_{a, l}\left(\varphi_{n}\right) \mathfrak{f}_{l}^{\mathrm{w}}\left(\varphi_{n}\right), \quad \forall f \in \mathcal{F}_{\phi}^{\mathrm{w}}, f \rightarrow l \in \mathcal{L}_{\phi}^{\mathrm{a}} .(5)
$$

where $\hat{p}_{a, l}\left(\varphi_{n}\right)$ represents the probability of entering a loading condition $l \in \mathcal{L}_{\phi}^{\mathrm{a}}$. The extension towards vectors $\hat{\boldsymbol{p}}_{l}(t)$ and $\mathfrak{f}_{l}\left(\varphi_{l}\right)$ is considered to be trivial and left to the reader.

Through this per-unit-degradation approach, the pdf of all failures are described with respect to the normal operation time $\varphi_{n}$ for each cohort $a$. Consequently, the overall survival function $R_{a}\left(\varphi_{n}\right)$ in the normal operation state $\left(n, \varphi_{n}\right)$ along a cohort $a$ may be described by

$$
R_{a}\left(\varphi_{n}\right)=\max \left(0.0,1.0-\sum_{f \in \mathcal{F}_{\phi}} \int_{0}^{\varphi_{n}} \mathfrak{f}_{a, f}\left(\varphi_{x}\right) \mathrm{d} \varphi_{x}\right) .
$$

\footnotetext{
${ }^{3}$ The presented expected wear-out is under the assumption that $\mathrm{f}^{\mathrm{w}}(\alpha)$ follows a Weibull distribution with a scale parameter $\nu$ equal to 10.0. A more comprehensive model for the expected lifetime of paper insulated transformers is introduced in the numerical illustration of this paper.
} 
Given that the sum of the integrals of all $\mathfrak{f}_{a, f}\left(\varphi_{n}\right)$ may exceed one, (6) is extended with the maximum operator in order to ensure that it is non-negative. Using the latter, the failure rates as given by (1) may be described as follows:

$$
\lambda_{a, f}\left(\varphi_{n} ; L\right)=\mathfrak{f}_{a, f}\left(\varphi_{n}\right) / R_{a}\left(\varphi_{n}\right), \quad f \in \mathcal{F}_{\phi} .
$$

\section{Effects on Future Failure Rates}

The approach outlined in the previous subsection only captures the effects of different loading conditions on the instantaneous failure rate. It does not yet capture the degradation effects following a nondetrimental loading condition and the associated impact on future failure rates. Concretely, the model in the previous subsection assumed that all degradation effects due to an abnormal loading condition subside once the component leaves that loading condition. To capture irreversible degradation effects, a time-inhomogeneous Markov chain is introduced in this subsection.

Under the condition of stochastic degradation, the degradation $\alpha$ of a component at any time instance $\left(t, \varphi_{n}\right)$ becomes a stochastic variable, and no longer equals the normal operation state sojourn time $\varphi_{n}$ at a time $t$. Certain failures $f \in \mathcal{F}_{\phi}^{\mathrm{w}} \subset$ $\mathcal{F}_{\phi}$, e.g., wear-out failures, may depend on a component's degradation $\alpha$ rather than its operation time $\varphi_{n}$. Stochastic degradation $\alpha$ along a cohort $a$ may be approximated through a time-inhomogeneous Markov chain under the following conditions:

1) all degradation drivers of a certain loading state, e.g., temperature changes in the component, subside immediately back to normal directly after leaving that state; and

2) a component having undergone minimal maintenance adopts the degradation variable of its destination cohort. The former essentially guarantees the Markov property, and is appropriate given highly available components, i.e., if rapid subsequent occurrence of stress operation is rare. The latter allows decoupling of the degradation processes along the individual cohorts, and seems appropriate given low temporal variation of the failure rates within the minimal maintenance time constant. The usage of a time-inhomogeneous Markov chain becomes less appropriate when the principal component becomes less reliable. At such a point, it may become necessary to apply stochastic process which do not require the memoryless property to model the remnant temperature effects. However, stochastic models which include memory are significantly less tractable. Given that the chosen stochastic process needs to be solved for each cohort $a$ and network components are generally highly reliable [23], a timeinhomogeneous Markov chain is the appropriate choice for the purpose of this paper.

A time-inhomogeneous Markov chain $\left\{A_{x}: x \geq 0\right\}$ is defined for each cohort $a$ (Fig. 3). The random variable $A_{x}$ describes a discrete state-space of component degradation states $\alpha_{i}$ and failure trapping states $\alpha_{f}$ at a discrete normal operation sojourn time $x \mathrm{~d} \varphi_{n}$. Following the Markov property, any transition within a timeframe $\mathrm{d} \varphi_{n}$ from one discrete degradation state $\alpha_{i}$ to another degradation state or a failure state $\alpha_{j}$, where $j \in \mathcal{J}=\mathcal{I} \cup \mathcal{F}_{\phi}$, is described by

$$
p_{i, j}=\mathbb{P}\left(A_{x+1}=\alpha_{j} \mid A_{x}=\alpha_{i}\right) .
$$

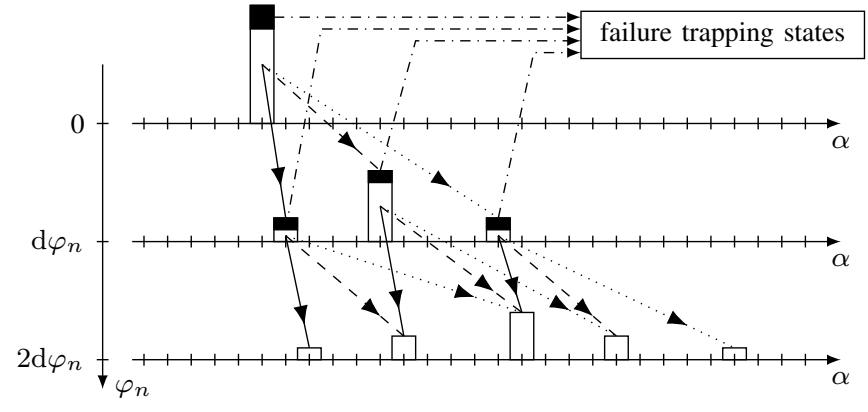

Fig. 3. Illustration of the Markov chain approximating a component's degradation $\alpha$ along a cohort $a$. Four possible transitions may occur: decreased ( failures $(-\cdot \cdot \cdot \cdot)$.

For a given degradation state $\alpha_{i}$ at an index $x$, there are two unknowns for each transition, i.e., $\alpha_{j}$ and $p_{i, j}$, depending on the transition type: failure or degradation transition.

A failure transition occurs when a component fails within a timeframe $\mathrm{d} \varphi_{n}$ due to a specific failure $f$, resulting in a transition to the corresponding failure trapping state $\alpha_{f}$ (9). The probability associated with such a transition is the conditional probability $\lambda_{a, f}=\mathfrak{f}_{a, f} / R_{a}$ over a time-period $\mathrm{d} \varphi_{n}$, and is evaluated using either $\alpha_{i}$ and/or $x \mathrm{~d} \varphi_{n}$, depending on the failure type (10)-(11). The failure pdf $\mathfrak{f}_{a, f}$ of the wear-out failures $f \in \mathcal{F}_{\phi}^{\mathrm{w}}$ is determined by the per-unitdegradation method described in the previous section. The survival function $R_{a}$ is evaluated at each node $i \in \mathcal{I}$ based on the complement of the sum of all failure trapping state probabilities:

$$
\begin{array}{lll}
\alpha_{j}=\alpha_{f}, & \forall i \in \mathcal{I}, f \in \mathcal{F}_{\phi}, \\
p_{i, j}=\lambda_{a, f}\left(\alpha_{i}, x \mathrm{~d} \varphi_{n}\right) \mathrm{d} \varphi_{n}, & \forall i \in \mathcal{I}, f \in \mathcal{F}_{\phi}^{\mathrm{w}}, \\
p_{i, j}=\lambda_{a, f}\left(x \mathrm{~d} \varphi_{n}\right) \mathrm{d} \varphi_{n}, & & \forall i \in \mathcal{I}, f \in \mathcal{F}_{\phi} / \mathcal{F}_{\phi}^{\mathrm{w}} .
\end{array}
$$

A degradation transition with a timeframe $\mathrm{d} \varphi_{n}$ occurs when the component does not fail and it was in a specific loading state $l$. Depending on the loading condition, i.e., normal or abnormal, the degradation state to which it transitions and associated probability are respectively determined through (12)(13) or (14)-(15).

$$
\begin{array}{rlrl}
\alpha_{j} & =\alpha_{i}+1, & & \forall i \in \mathcal{I}, l \in \mathcal{L}_{\phi}^{\mathrm{n}}, \\
p_{i, j}=\left(1-\sum_{f \in \mathcal{F}_{\phi}} p_{i, f}\right) p_{a, l}\left(x \mathrm{~d} \varphi_{n}\right), & & \forall i \in \mathcal{I}, l \in \mathcal{L}_{\phi}^{\mathrm{n}}, \\
\alpha_{j}=\alpha_{i}+\left\lceil\left(\mathbb{E}\left(\Delta \alpha_{l}^{\mathrm{rel}}\right) / \mathrm{d} \varphi_{n}\right\rceil,\right. & & \forall i \in \mathcal{I}, l \in \mathcal{L}_{\phi}^{\mathrm{a}}, \\
p_{i, j}=\left(1-\sum_{f \in \mathcal{F}_{\phi}} p_{i, f}\right) \hat{p}_{a, l}\left(x \mathrm{~d} \varphi_{n}\right), & & \forall i \in \mathcal{I}, l \in \mathcal{L}_{\phi}^{\mathrm{a}} .
\end{array}
$$

The degradation state $\alpha_{j}$ to which a component transitions after an abnormal loading condition is determined similarly to the previous subsection, i.e., using the expected relative degradation $\mathbb{E}\left(\Delta \alpha_{l}^{\text {rel }}\right)$ associated with an abnormal loading condition $l \in \mathcal{L}_{\phi}^{\mathrm{a}}$. The corresponding transition probability $p_{i, j}$ equals the combination of the probability of not failing in a timeframe $d \varphi_{n}$, i.e., the complement of the sum of all failure transitions probabilities and the probability of entering the corresponding loading state $\hat{p}_{a, l}\left(x \mathrm{~d} \varphi_{n}\right)$. 


\section{NUMERICAL ILLUSTRATION}

This section introduces a numerical illustration with the aim of demonstrating the effects of redundancy dependence on the unavailability of transformers. To this end, a test system $s_{t}$ is introduced consisting of two transformers, where one provides redundancy upon the failure of the other. Realistic transformer data is considered to model the effects of load changes on both its instantaneous and future failure rates. The example of thermal degradation of transformers is used for this numerical illustration, however, similar models exist for a number of components, e.g., polymer degradation of cables [24], corrosion of steel pipes [25], etc.

\section{A. Test System}

The test system $s_{t}$ consists of two transformers $\phi_{1}$ and $\phi_{2}$ (Fig. 4b). A transformer has two protection zones; initially, a fault should be cleared by breakers $b^{\text {hv }}$ and $b^{\text {lv }}$; if unsuccessful, the fault is isolated by breaker $b^{\text {sv }}$. Whenever the fault is cleared by the second protection zone breaker, the topology is manually altered to a state similar to where the fault was cleared by the first protection zone breakers. Following the latter or following direct clearing by the first protection zone breakers, the failed transformer is maintained and consequently restored to a working condition. If available, i.e., in state $\left(n, \varphi_{n}\right)$, transformer $\phi_{2}$ provides redundancy for transformer $\phi_{1}$ by closing breaker $b^{\mathrm{r}}$, if the latter enters any state $\left(r, \varphi_{r}\right)_{e \in \mathcal{F}_{\phi_{1}}}$.

The stochastic behavior of both transformers is modeled by a state-transition diagram consisting of four state types (Fig. 4a and 4a). Each post-failure state type has a separate state for each possible failure. Table II gives an overview of the transitions between the different states. Transformer $\phi_{1}$ is subject to three failures $\left\{e_{1}, e_{2}, e_{3}\right\} \in \mathcal{F}_{\phi_{1}}$, respectively with a weight $p_{e}$ of $0.2,0.1$ and 0.7 , and $a_{e}^{\chi}$ equal to $0.2,1.0$ and 2.0. The third failure of transformer $\phi_{1}$ is modeled by a Weibull distribution whereas all others are described by an exponential distribution. The first two failures require minimal corrective maintenance whereas the third failure requires perfect corrective maintenance. The scale parameter $b_{e}^{\chi}$ of the maintenance actions are respectively equal to 1.0, 2.5 and 5.0. All other transitions are fully described by Table II.

Transformer $\phi_{2}$ is subject to two failures $\left\{f_{1}, f_{2}\right\}=\mathcal{F}_{\phi_{2}}$, where the latter is a wear-out failure depending on its loading state: $\left\{f_{2}\right\} \in \mathcal{F}_{\phi_{2}}^{\mathrm{w}}$. Transformer $\phi_{2}$ has two loading states $\left\{l_{1}, l_{2}\right\} \in \mathcal{L}_{\phi_{2}}$. The first loading state $l_{1} \in \mathcal{L}_{\phi_{2}}^{\mathrm{n}}$ is the normal loading state $\left(\mathrm{I}_{l_{1}}^{\mathrm{pu}}=0.75\right)$. Loading state $l_{1}$ is
TABLE II

TRANSITION TYPES FOR THE STATE-TRANSITION DIAGRAM (FIG. 4A AND 4C), WHERE $p, \chi$ AND $\nu$ ARE THE WEIGHT, SCALE AND SHAPE.

\begin{tabular}{cllccc} 
Sym. & Transition type & Distr. Type & $p[-]$ & $\chi[\mathrm{h}]$ & $\nu[-]$ \\
\hline$\lambda$ & Failure & Exp./Weibull & $p_{e} / 1$ & $a_{e / f}^{\chi} \cdot 10^{5}$ & 10 \\
$\xi^{\mathrm{P}}$ & Primary fault clearing & Dirac $(100 \mathrm{~ms})$ & 0.8 & - & - \\
$\xi^{\mathrm{S}}$ & Secondary fault clearing & Dirac $(350 \mathrm{~ms})$ & 0.2 & - & - \\
$\xi^{\mathrm{R}}$ & Topological action & Log-normal & 1.0 & $10^{1}$ & 0.08 \\
$\mu$ & Maintainability action & Weibull & 1.0 & $b_{e / f}^{\chi} \cdot 10^{2}$ & 10 \\
\hline
\end{tabular}

the active loading state whenever transformer $\phi_{1}$ is not in a state $\left(r, \varphi_{r}\right)_{e \in \mathcal{F}_{\phi_{1}}}$ (Fig. 4a). The second loading state $l_{2} \in \mathcal{L}_{\phi_{2}}^{\mathrm{a}}$ is an abnormal loading state $\left(\mathrm{I}_{l_{2}}^{\mathrm{pu}}=1.50\right)$ and corresponds with any state $\left(r, \varphi_{r}\right)_{e \in \mathcal{F}_{\phi_{1}}}$ (Fig. $4 \mathrm{a}$ ). The random failure $f_{1}$ is modeled by an exponential distribution with a scale parameter equal to $2.0 \cdot 10^{5} \mathrm{~h} \approx 22.5 \mathrm{yr}$. Failure $f_{2}$ is described by a Weibull distribution with a shape parameter $\nu$ of either (a) 1.0, (b) 3.0 or (c) 5.0. Note that a Weibull distribution with a shape parameter $\nu$ equal to one reduces to an exponential distribution. The scale parameter $\chi$ of a Weibull distribution correlates with the expected lifetime $\mathbb{E}\left(\Phi_{n}\right)$ under a certain loading condition through

$$
\chi=\mathbb{E}\left(\Phi_{n}\right) / \Gamma(1+1 / \nu),
$$

where $\Gamma$ represents the gamma function. The expected lifetime $\mathbb{E}\left(\Phi_{n}\right)$ of a transformer is mainly dependent on the degradation of its insulation material brought on by thermal stress, i.e., its loading condition $\mathrm{I}^{\mathrm{pu}}$. The winding hot-spot temperature is generally regarded as the principal indicator for the latter. Specifically, the IEC 60354 standard states that a normal paper insulated transformer has an expected lifetime of 25 years with a winding hot-spot temperature $\theta^{\text {hs }}$ of $98^{\circ} \mathrm{C}$ [3]. The modified IEEE model presented in [26] describes the dynamic hot-spot temperature of a transformer as follows

$$
\begin{aligned}
\tau^{\text {to }} \frac{\mathrm{d} \theta^{\text {to }}}{\mathrm{d} t} & =-\theta^{\text {to }}+\theta^{\mathrm{a}}+\Delta \theta^{\text {to }}, \\
\tau^{\mathrm{w}} \frac{\mathrm{d} \theta^{\mathrm{hs}}}{\mathrm{d} t} & =-\theta^{\mathrm{hs}}+\theta^{\text {to }}+\Delta \theta^{\mathrm{hs}}, \\
\Delta \theta^{\text {to }} & =\Delta \theta^{\text {to,r }} \cdot\left[\frac{\left(\mathrm{I}^{\mathrm{pu}}\right)^{2} R+1}{R+1}\right]^{n}, \\
\Delta \theta^{\mathrm{hs}} & =\Delta \theta^{\mathrm{hs}, \mathrm{r}} \cdot\left(\mathrm{I}^{\mathrm{pu}}\right)^{2 n},
\end{aligned}
$$

where: $\theta^{\mathrm{a}}$

$\tau^{\text {to }} \quad$ top-oil time constant

$\tau^{w} \quad$ winding time constant

$\begin{aligned} & \phi_{2} \\ & 25.0{ }^{\circ} \mathrm{C} \\ & 150.0 \mathrm{~h} \\ & 10.0 \mathrm{~min}\end{aligned}$

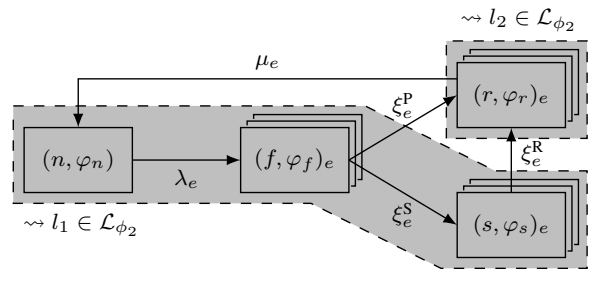

(a) state-transition diagram transformer $\phi_{1}$

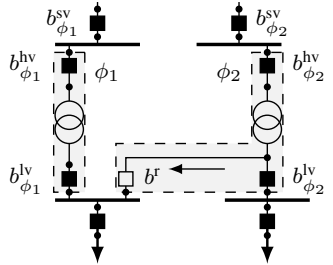

(b) test system $s_{t}$

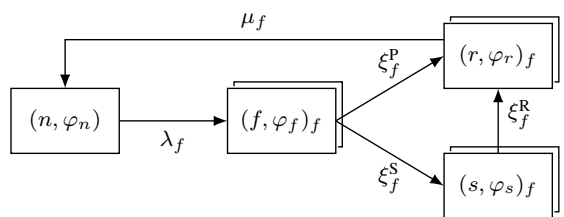

(c) state-transition diagram transformer $\phi_{2}$

Fig. 4. Test system $s_{t}(4 \mathrm{~b})$ and the corresponding state-transition diagrams (4a) and (4c) of transformers $\phi_{1}$ and $\phi_{2}$. The former is annotated to show $\mathcal{L}_{\phi_{2}}$. 


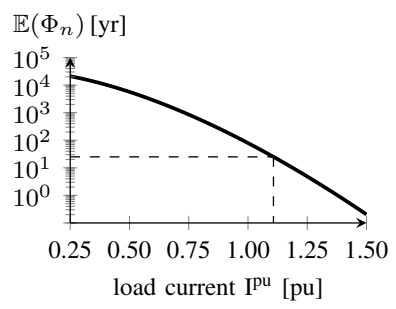

(a) $\mathbb{E}\left(\Phi_{n}\right)(25 \mathrm{yr}:-$ - )

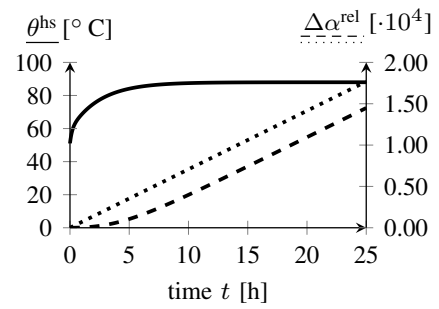

(b) dynamic $\theta^{\text {hs }}$ and $\Delta \alpha^{\text {rel }}$

Fig. 5. The expected lifetime $\mathbb{E}\left(\Phi_{n}\right)$ of transformer $\phi_{2}$ (Fig. 5a) and its hot-spot temperature $\theta^{\text {hs }}$ and relative degradation $\Delta \alpha^{\text {rel }}$ (Fig. 5b).

$\begin{array}{llc}\Delta \theta^{\mathrm{to}, \mathrm{r}} & \text { rated top-oil temp. rise over } \theta^{\mathrm{a}} & 48.0{ }^{\circ} \mathrm{C} \\ \Delta \theta^{\mathrm{hs}, \mathrm{r}} & \text { rated hot-spot temp. rise over } \theta^{\text {to }} & 15.0{ }^{\circ} \mathrm{C} \\ R & \text { ratio of load to no-load losses } & 6.0 \\ n & \text { exponent defining non-linearity } & 0.8\end{array}$

Based on the hot-spot temperature $\theta^{\text {hs }}$, the relative degradation function $\Delta \alpha^{\text {rel }}$ of a normal paper insulated transformer can be written as

$$
\Delta \alpha^{\mathrm{rel}}=2^{\left(\theta^{\mathrm{hs}}-98\right) / 6} .
$$

Using the latter, the expected lifetime $\mathbb{E}\left(\Phi_{n}\right)$ of a normal paper insulated transformer can be determined for any loading condition $\mathrm{I}^{\mathrm{pu}}$ (Fig. 5a). Furthermore, this model enables assessment of the dynamic hot-spot temperature $\theta^{\text {hs }}$ following a change in transformer loading, e.g., $\mathrm{I}^{\mathrm{pu}}: 0.75 \rightarrow 1.50$ (full line in Fig. 5b). Using this dynamic hot-spot temperature, the relative degradation $\Delta \alpha^{\text {rel }}$ is determined for the abnormal loading state $l_{2}$ compared to the normal loading state $l_{1}$ (dashed line in Fig. 5b). Erroneous usage of the steady state hot-spot temperature may lead to a significant error when assessing the relative degradation of the transformer (dotted line in Fig. 5b). For the presented example, the relative error would still approximately be $4.5 \%$ after $100 \mathrm{~h}$. Using the presented models and data, both the effect on the instantaneous and future failure rates of transformer $\phi_{2}$ may be determined for any loading scenario. Both failures $\left\{f_{1}, f_{2}\right\} \in \mathcal{F}_{\phi_{2}}$ require perfect corrective maintenance, respectively modeled with a scale parameter $b_{f}^{\chi}$ equal to 1.0 and 5.0. All other transitions are fully described by Table II.

\section{B. Results and Discussion}

Transformers $\phi_{1}$ and $\phi_{2}$ are sequentially assessed, as the latter is dependent on the former, using the method presented in [19] over a time period of twenty years with a timestep $\mathrm{d} a$ of one day. The resulting unavailability and failure probabilities of transformer $\phi_{1}$ are respectively depicted in Figures $6 \mathrm{a}$ and $6 \mathrm{~b}$. Notice that the extremums of the failure probabilities are in the same order, but their resulting effect on the unavailability differ significantly given the difference in duration of their respective maintenance action. The reliability of transformer $\phi_{2}$ depends on its loading throughout the considered time period of twenty years. To this end, the loading scenario $\mathrm{I}^{\mathrm{pu}}: 0.75 \rightarrow 1.50$ is evaluated, with (w) and without (w/o) accounting for transformer degradation, i.e., the Markov model presented in Section II-B. In its normal

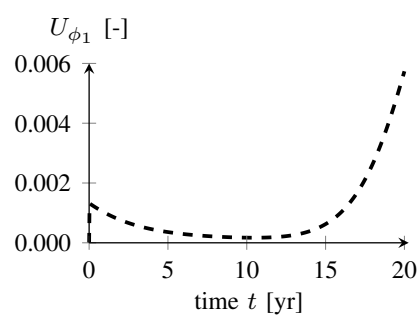

(a) component unavailability $U_{\phi}(t)$

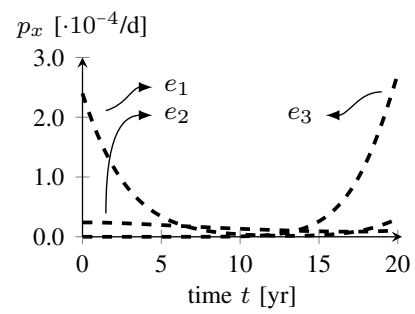

(b) failure probabilities $p_{e}(t)$
Fig. 6. Results of the availability assessment of transformer $\phi_{1}$ over a time period of twenty years with a time-step $\mathrm{d} a$ of one day.

loading state $l_{1}$, i.e., $\mathrm{I}_{l_{1}}^{\mathrm{pu}}=0.75$, the transformer $\phi_{2}$ has an expected wear-out operation time $\mathbb{E}\left(\Phi_{n}\right)$ of $855.1 \mathrm{yr}$. Given that the abnormal loading state $l_{2}$ corresponds with three different states $\left\{\left(r, \varphi_{r}\right)_{e_{1}},\left(r, \varphi_{r}\right)_{e_{2}},\left(r, \varphi_{r}\right)_{e_{3}}\right\}$ of transformer $\phi_{1}$, each with a distinct pdf $\mathfrak{f}_{\mu_{e}}\left(\varphi_{r}\right)$, the corresponding expected degradation $\mathbb{E}\left(\Delta \alpha^{\text {rel }}\right)$ of transformer $\phi_{2}$ respectively equals $42.7,111.2$ and $225.3 \mathrm{yr}$. Each of these expected degradation values corresponds with their respective operation state entry probability $\hat{p}_{r}(t)$.

Figure 7 shows the wear-out failure probabilities $p_{f_{2}}(t)$ for the different shape parameters: 1.0, 3.0 and 5.0, both with (w) or without (w/o) accounting for degradation. The random failure probability $p_{f_{1}}(t)$ is also included as a reference (dashdotted in Fig. 7). Several takeaways may be pointed out:

1) The dynamic behavior of the failure probability $p_{f_{2}}(t)$ follows that of its principal transformer's unavailability $U_{\phi_{1}}(t)$ (Fig. 6a).

2) For the exponential case $(\nu=1.0)$, it can be seen that accounting for degradation has no influence on the failure probability $p_{f_{2}}(t)$, i.e., (w)- and (w/o)-curves overlap. This is the direct result of the corresponding failure rate being constant, or in other words independent of the component's degradation. Consequently, both curves (w) and (w/o) overlapping for the exponential case validates the presented approach under these conditions.

3) As the shape parameter $\nu$ deviates further from one, the relative error related to not accounting for degradation increases: $\mathrm{L}^{2}=0.603 \rightarrow 3.678$ for $\nu: 3.0 \rightarrow 5.0$.

Consequently, the differences in failure probability are also reflected in the system unavailability $U_{s_{t}}(t)$ (Fig. 8).

\section{CONCLUSION}

This paper presented a holistic approach consisting of two models to describe redundancy dependence for semicontinuous MSS accounting for (i) competing risks, (ii) general failure rates, and (iii) dynamic degradation. First, a per-unitdegradation method was introduced to model the accelerated degradation during different loading conditions within the context of competing risks. Second, a time-inhomogeneous Markov chain is introduced to assess the accumulated degradation following a nondetrimental abnormal loading condition. The numerical illustration shows the necessity of both models to determine the effects of redundancy dependence, specifi- 


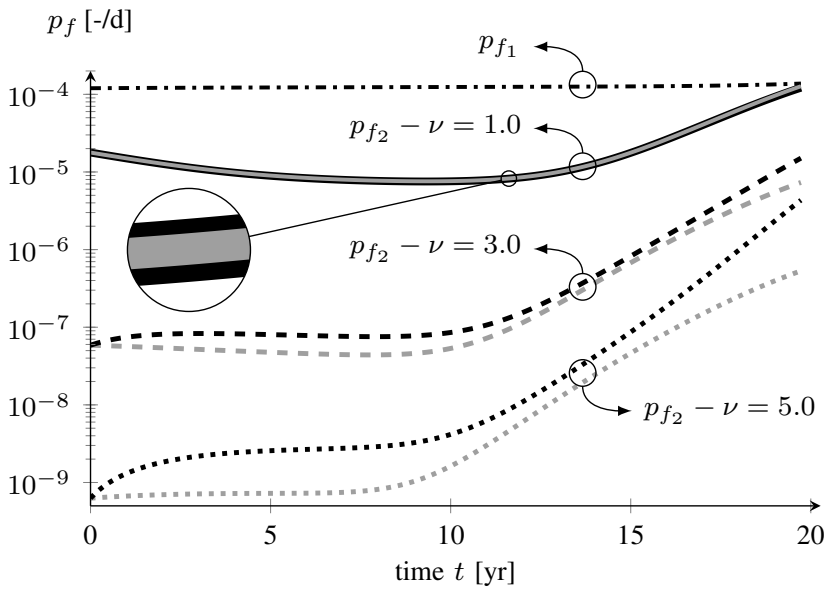

Fig. 7. Failure probabilities $p_{f_{1}}(t)(-\cdot-)$ and $p_{f_{2}}(t)$ of transformer $\phi_{2}$ for scenarios $\mathrm{I}^{\mathrm{pu}}: 0.75 \rightarrow 1.50$ (w/o) in gray and (w) in black, where failure $f_{2}$ follows a Weibull distribution with a shape parameter $\nu$ of (a) 1.0 ( - ), (b) $3.0(--)$ and (c) $5.0(\cdots)$.

cally when the underlying pdf of the wear-out failure has a shape parameter which exceeds 1.0.

\section{REFERENCES}

[1] E. Zio, "Reliability engineering: Old problems and new challenges," Reliab. Eng. Syst. Saf., vol. 94, no. 2, pp. 125-141, 2009.

[2] International Electrotechnical Commission (IEC), "Degradation, < of an item >," 2015. [Online]. Available: http://www.electropedia.org/iev/ iev.nsf/display?openform\&ievref=192-01-20 [Accessed: May 15, 2020]

[3] J. Schlabbach and K. H. Rofalski, "Transformers," in Power Syst. Eng., 1st ed. Weinheim: Wiley-VCH, 2008, ch. 7, pp. 81-109.

[4] S. Kotz, C. D. Lai, and M. Xie, "On the effect of redundancy for systems with dependent components," IIE Trans., vol. 35, no. 12, pp. 1103-1110, 2003.

[5] A. Blokus, "Reliability analysis of large systems with dependent components," Int. J. Reliab. Qual. Saf. Eng., vol. 13, no. 1, pp. 1-14, 2006.

[6] H. Yu, C. Chu, E. Châtelet, and F. Yalaoui, "Reliability optimization of a redundant system with failure dependencies," Reliab. Eng. Syst. Saf., vol. 92, no. 12, pp. 1627-1634, 2007.

[7] V. da Costa Bueno, "Minimal standby redundancy allocation in a k-outof-n:F system of dependent components," Eur. J. Oper. Res., vol. 165, no. 3, pp. 786-793, 2005

[8] V. da Costa Bueno and I. Martins do Carmo, "Active redundancy allocation for a k-out-of-n:F system of dependent components," Eur. J. Oper. Res., vol. 176, no. 2, pp. 1041-1051, 2007.

[9] F. Belzunce, H. Martínez-Puertas, and J. M. Ruiz, "On allocation of redundant components for systems with dependent components," Eur. J. Oper. Res., vol. 230, no. 3, pp. 573-580, 2013.

[10] H. Yu, C. Chu, and É. Châtelet, "Availability optimization of a redundant system through dependency modeling," Appl. Math. Model., vol. 38, no. 19-20, pp. 4574-4585, 2014.

[11] L. Yuan, "Reliability analysis for a k-out-of-n:G system with redundant dependency and repairmen having multiple vacations," Appl. Math. Comput., vol. 218, no. 24, pp. 11959-11969, 2012.

[12] I. Norros, P. Kuusela, J. Lapuyade-Lahorgue, M. Naldi, and M. Sirviö, "Repairable systems with dependent components: stochastic process techniques and models," IEEE Trans. Reliab., vol. 63, no. 4, pp. 899912, 2014

[13] H. Jia, Y. Ding, R. Peng, and Y. Song, "Reliability evaluation for demand-based warm standby systems considering degradation process," IEEE Trans. Reliab., vol. 66, no. 3, pp. 795-805, 2017.

[14] M. Xu, X. Jin, S. Kamarthi, and M. Noor-E-Alam, "A failuredependency modeling and state discretization approach for conditionbased maintenance optimization of multi-component systems," J. Manuf. Syst., vol. 47, pp. 141-152, 2018.

[15] Y. Sun, L. Ma, J. Mathew, and S. Zhang, "An analytical model for interactive failures," Reliab. Eng. Syst. Saf., vol. 91, no. 5, pp. 495-504, 2006.

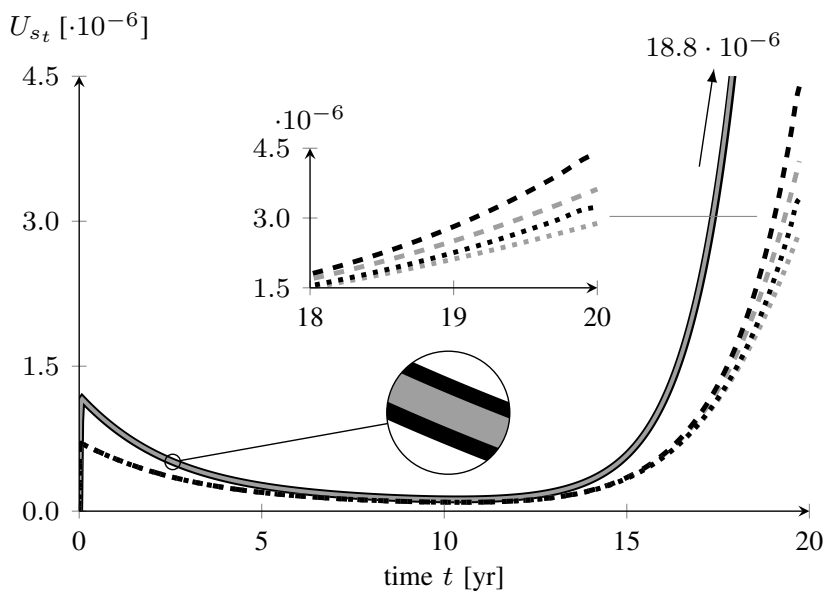

Fig. 8. System unavailability $U_{s_{t}}(t)$ for scenarios $\mathrm{I}^{\mathrm{pu}}: 0.75 \rightarrow 1.50(\mathrm{w} / \mathrm{o})$ in gray and $(w)$ in black, where failure $f_{2}$ follows a Weibull distribution with a shape parameter $\nu$ of (a) $1.0(-$ ), (b) $3.0(--)$ and (c) $5.0(\ldots)$ ).

[16] L. Wang, J. Xujie, and J. Zhang, "Reliability evaluation for multistate Markov repairable systems with redundant dependencies," Qual. Technol. Quant. Manag., vol. 10, no. 3, pp. 277-289, 2013.

[17] N. C. Caballé, I. T. Castro, C. J. Pérez, and J. M. Lanza-Gutiérrez, "A condition-based maintenance of a dependent degradation-thresholdshock model in a system with multiple degradation processes," Reliab. Eng. Syst. Saf., vol. 134, pp. 98-109, 2015.

[18] M. Fan, Z. Zeng, E. Zio, and R. Kang, "Modeling dependent competing failure processes with degradation-shock dependence," Reliab. Eng. Syst. Saf., vol. 165, no. June 2016, pp. 422-430, 2017.

[19] T. Van Acker and D. Van Hertem, "Stochastic process for the availability assessment of single-feeder industrial energy system sections," IEEE Trans. Reliab., vol. 67, no. 4, pp. 1459-1467, 2018.

[20] T. Aven and U. Jensen, Stochastic Models in Reliability, 2nd ed. New York, NY: Springer, 2013.

[21] O. Angulo, J. C. López-Marcos, M. A. López-Marcos, and F. A. Milner, "A numerical method for nonlinear age-structured population models with finite maximum age," J. Math. Anal. Appl., vol. 361, no. 1, pp. $150-160,2010$

[22] J. D. Kalbfleisch and R. L. Prentice, "Competing Risks and Multistate Models," in Stat. Anal. Fail. Time Data, 2nd ed. Hoboken, NJ: WileyInterscience, 2002, ch. 8, pp. 247-277.

[23] Y. He, "Reliability of distribution network components," in CIRED Int. Conf. Electr. Distrib., 2009, pp. 8-11.

[24] N. Bowler and S. Liu, "Aging mechanisms and monitoring of cable polymers," Int. J. Progn. Heal. Manag., vol. 6, no. Special Issue Nuclear Energy PHM, 2015.

[25] M. Hagarová, J. Cervová, and M. Vojtko, "Corrosion degradation of steel pipes in indirect cooling circuit of gas cleaning," Mater. Sci. Forum, vol. 811 , pp. $41-48,2015$

[26] IEEE Power and Energy Society, "IEEE guide for loading mineral-oilimmersed transformers and step-voltage regulators," 2012.

[27] R. Gono, M. Kratky, and S. Rusek, "Reliability of Distribution Network Components based on Failure Databases," AASRI Procedia, vol. 2, pp. 75-80, 2012.

Tom Van Acker (S'14-M'20) received the M.Eng., M.Sc. and Ph.D. degrees from KU Leuven, Belgium in 2012, 2014 and 2020, respectively. Currently, he is a post-doctoral researcher at KU Leuven. His main areas of research interest are stochastic processes and optimization in a power system context. 
Dirk Van Hertem (S'02-SM'09) received the M.Eng. degree in 2001 from the Katholieke Hogeschool Kempen, Geel, Belgium, and the M.Sc. degree in electrical engineering and the Ph.D. degree from KU Leuven, Leuven, Belgium, in 2003 and 2009, respectively. In 2010, He was a member of EPS group at the Royal Institute of Technology (KTH), Stockholm. Since spring 2011, he has been with KU Leuven, where he is an Associate Professor in the ELECTA group. He has authored/coauthored more than 200 scientific works in international conferences and journals. His special fields of interest are decision support for grid operators, power system operation and control in systems with FACTS and HVdc, and building the transmission system of the future, including offshore grids and the supergrid concept. He is an active member of both IEEE (PES and IAS) and Cigré. He is currently the Chair of the steering committee of the ISGT Europe conference. He was the General Chair of both the IEEE EnergyCon 2016 conference, held in Leuven, and the IEEE summit on sustainability - vision on 2050 . 